\title{
Plasmon spectroscopy of free-standing graphene films
}

\author{
T. Eberlein, ${ }^{1}$ U. Bangert, ${ }^{2}$ R. R. Nair, ${ }^{2,4}$ R. Jones, ${ }^{1}$ M. Gass, ${ }^{3}$ A. L. Bleloch,${ }^{3}$ K. S. Novoselov, ${ }^{4}$ \\ A. Geim, ${ }^{4}$ and P. R. Briddon ${ }^{5}$ \\ ${ }^{1}$ School of Physics, University of Exeter, Stocker Road, Exeter, EX4 4QL, United Kingdom \\ ${ }^{2}$ School of Materials, The University of Manchester, Manchester M1 7HS, United Kingdom \\ ${ }^{3}$ SuperSTEM Laboratories, CCLRC Daresbury Laboratory, Warrington WA4 4AD, United Kingdom \\ ${ }^{4}$ School of Physics and Astronomy, The University of Manchester, Manchester M1 7HS, United Kingdom \\ ${ }^{5}$ School of Natural Sciences, University of Newcastle upon Tyne, Newcastle upon Tyne NE1 7RU, United Kingdom
}

(Received 5 November 2007; revised manuscript received 25 January 2008; published 18 June 2008)

\begin{abstract}
Plasmon spectroscopy of the thinnest possible membrane, a single layer of carbon atoms: graphene, has been carried out in conjunction with ab initio calculations of the low loss function. We observe $\pi$ and $\pi+\sigma$-surface plasmon modes in free-standing single sheets at 4.7 and $14.6 \mathrm{eV}$, which are substantially redshifted from their values in graphite. These modes are in very good agreement with the theoretical spectra, which find the $\pi$ - and $\pi+\sigma$ in-plane modes of graphene at 4.8 and $14.5 \mathrm{eV}$. We also find that there is little loss caused by out-of-plane modes for energies less than about $10 \mathrm{eV}$.
\end{abstract}

DOI: 10.1103/PhysRevB.77.233406

There has recently been intense interest in the properties of graphene $e^{1,2}$ and, in particular, those properties that distinguish it from graphite. Among the many suggested areas in which graphene may excel is, e.g., its application for sensors due to the sensitivity of its electronic structure to adsorbates. ${ }^{3}$ Low loss energy electron spectroscopy provides a way of detecting changes in the electronic structure, which are highly spatially resolved. To be able to employ this technique, the loss spectra of graphene and graphite must be clearly understood.

From an experimental viewpoint, an essential task in the research into two-dimensional (2D) structures is to provide evidence that they do indeed exist. This is especially important because theory does not allow the existence of perfect crystals in 2D space. The most conclusive evidence for the existence of free-standing graphene has so far been obtained from electron diffraction experiments ${ }^{4}$ and all previously published high resolution electron microscopy (HREM) images, to our knowledge, are of bilayers. By carrying out highly spatially resolved electron energy loss spectroscopy (EELS), we observe specific redshifts in the frequency of plasmons in sample positions concomitant with single graphene sheets, very similar to those observed in singlewall carbon nanotubes (SWCNTs). ${ }^{5}$ We also find further evidence for existence of a single sheet by combining optical and high angle annular dark field (HAADF) imaging.

Surface plasmon behavior in thin metal sheets is well documented experimentally and explained using dielectric theory. ${ }^{6,7}$ There are also numerous reports on surface plasmons in graphite and carbon nanotubes. ${ }^{5,8-11}$ Plasmon behavior of truly 2D graphite, i.e., of monolayer graphite foils has been theoretically suggested in a number of papers ${ }^{12,13}$ and has been experimentally studied for flat monolayers grown on TiC..$^{14}$ However, there has been, so far, no experimental study for free-standing sheets. There have, however, been studies of SWCNTs and much of the interpretation of plasmon behavior for SWCNTs for radius $r \rightarrow \infty$ can be applied to free-standing single graphene sheets, ${ }^{5}$ particularly as the tubes are free standing also. A characteristic of thin foils is the vanishing of the bulk plasmon mode, leaving only the surface plasmon mode; the out-of-plane and in-plane contri- butions of the latter split in energy when the product of thickness $d$ and momentum $q, d q \rightarrow 0$; in graphite the maximum and/or minimum energy of the $\pi \rightarrow \pi^{*}$ transition tends to roughly 7 and $5 \mathrm{eV}$ and of the $\sigma \rightarrow \sigma^{*}$ transition to 20 and $15 \mathrm{eV}$, respectively. ${ }^{5}$

The $\boldsymbol{E}$ field of a fast moving particle is elongated along its direction of travel, therefore, when passing perpendicularly through a graphene foil, mainly, the out-of plane mode with momentum $\hbar \boldsymbol{q}$ parallel to $\boldsymbol{E}$ should be excited. However, as shown below, these modes are forbidden in a single layer and they have weak intensity in graphite. In an EELS experiment carried out in a scanning transmission electron microscope (STEM), although the momentum transfer is close to zero, nonetheless, $\boldsymbol{q}$ has a considerable in-plane component because the collection angle is several millirad. For this reason, we will observe those $\pi$ surface and bulk plasmons excited with $\boldsymbol{q}$ parallel to $\boldsymbol{a}$.

Large graphene membranes were prepared by a micromechanical cleavage ${ }^{1}$ of natural graphite on top of an oxidized Si wafer. This deposition technique has the benefit of allowing a quick and easy identification of mono- and multiple layers by the additional optical contrast with respect to the oxidized wafer. By using photolithography, a perforated copper-gold film, serving as scaffold that could be used in standard transmission electron microcopy holders, was deposited on top of the graphene crystallites. This scaffold was lifted off the Si wafer, leaving the graphene attached to it. ${ }^{15}$ Prior to electron microscopy, optical microscopy was used to identify regions of one to few layers. HAADF images of one-, two-, and five-layer regions are shown in Fig. 1; the rectangular boxes show the regions in which the intensity [Fig. 1(d)], integrated over the width of the box, is traced. The heights of the contrast profiles are multiples of the smallest height measured in Fig. 1(a), confirming that the contrast in the dark region [Fig. 1(a)] arises from one single graphene sheet. Close inspection of the dark areas in each of the images reveals uniform contrast upon which the hexagonal atomic lattice can be seen. Filtering of the HAADF images enhances the visibility of the lattice [white-framed boxes in Figs. 1(a)-1(c)]. The images are proof that the dark areas are pristinely clean, i.e., free from contaminants. 


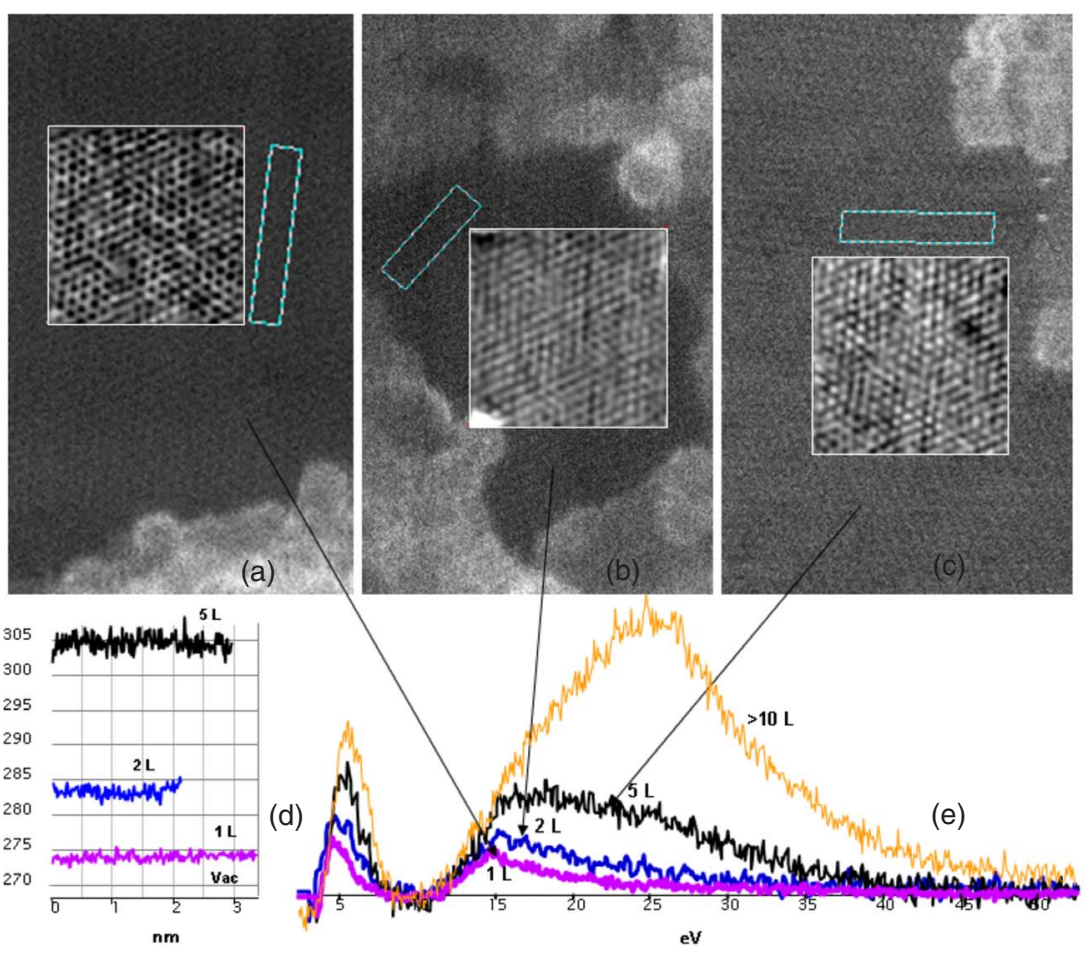

FIG. 1. (Color online) (a)-(c) HAADF STEM images of 'clean' patches revealing one, two, and five layers of graphene. The area in the white-framed boxes has been subjected to a low pass filter to disclose the atoms more clearly: In the single layer the atoms in the six rings are white and the hexagon center is black. (d) Intensity traces have been taken along the long dimensions of the rectangular cyan-framed boxes in (a)-(c); these traces were then averaged over 40 pixels (width of boxes). They show step-wise increase of the HAADF intensity corresponding to the layer number. The vacuum intensity is at $\sim 268$. (e) EEL spectra of one, two, five and several layers of graphene showing the $\pi$-and the $\pi+\sigma$ plasmon. The spectra are extracted from spectrum images; they are background subtracted and each summed over 25 pixels.
The Daresbury aberration corrected SuperSTEM with an Enfina EELS spectrometer was used for HREM bright field and HAADF imaging in conjunction with EELS. EELS of the plasmon region was carried out with a dispersion of 0.05 $\mathrm{eV}$ per channel at an energy resolution (full width at half maximum of the zero loss peak) of $0.3-0.4 \mathrm{eV}$. The collection semiangle was approximately $19 \mathrm{mrad}$. The operating voltage was $100 \mathrm{kV}$. In order to minimize beam damage, the acquisition time per EEL spectrum was kept short $(\sim 0.1 \mathrm{~s})$; the dose each pixel in a spectrum image received was $\sim 1.6 \times 10^{7}$ electrons. When the acquisition time was increased or when repeated scans were performed on the same area, electron beam damage became manifest in formation of point defects (single and multiple vacancies). ${ }^{16}$

In Fig. 1(e), we show raw EEL data obtained by subtracting the zero loss peak under identical conditions. The spectra were all taken under the same acquisition conditions, hence, direct comparison between them is possible.

The plasmon excitations in graphitic structures consist of the $\pi$ - and $\pi+\sigma$ plasmons both exhibiting bulk and surface modes. In Fig. 1(e), the plasmon spectra taken in clean patches of one, two, five and multiple sheets shows that the $\pi$-mode, at $7 \mathrm{eV}$ in graphite, has shifted to $4.7 \mathrm{eV}$ in the single layer. Furthermore, the spectrum here exhibits only the $\pi+\sigma$-surface mode at $14.6 \mathrm{eV}$ and the $26 \mathrm{eV}$ plasmon in graphite is not present. The shape and intensity of the onelayer plasmon structure was repeatedly measured in different places on the same sample, in different samples, and even in different experimental sessions. Given the same acquisition conditions (e.g., energy dispersion, electron beam current, and dwell time), nearly identical spectra were obtained, which, at the same time, constituted the lowest plasmon signal measured overall. For two sheets, the triangular shape of the $\pi+\sigma$ resonance shifts toward higher energies and the integrated intensity under the peak (between 10 and $40 \mathrm{eV}$ ) approximately doubles. A plateau between 15 and $20 \mathrm{eV}$ starts to appear, which becomes pronounced for increasing numbers of sheets: In the five-layer patch features above 15 $\mathrm{eV}$ start to appear, the plasmon maximum keeps moving to higher energies, accompanied by further broadening, and the integrated intensity increases to $\sim 5$ times the value of the single sheet. The plasmon structure for more than 10 sheets strongly resembles that of the graphite. The plasmon characteristics are, thus, supreme indicators for the presence of single layers. However, the smallest amount of contamination, e.g., caused by individual molecular adsorbates, introduces a "three-dimensional" component and leads to a breakdown of the $2 \mathrm{D}$ behavior, as is frequently evidenced. ${ }^{17}$ Contaminants can easily be discerned in HREM and HAADF images; in fact the whitish contrast bordering the clean patches in the HAADF images Figs. 1(a)-1(c) represent exactly this. We note that unlike low loss spectra, core loss spectra were not found appropriate to reveal characteristics that could help distinguish one layer from few layers by their shape. In the spectrum of a larger number of graphene sheets in Fig. 1(e), resembling much that of a thin graphitic slab, the well-known graphitic bulk $\pi$ - and $\sigma$-modes can be observed at 7 and $26 \mathrm{eV}$, respectively. We have consistently observed $\sigma$-plasmon behavior with distinct occurrence of higher energy components, accompanied by a step-wise increase in the HAADF intensity when going from one to two or more sheets. Values of the HAADF intensity for one, two, and more sheets [see Fig. 1(d)] were measured against the vacuum HAADF intensity and displayed increments of approximately integer multiples of the first increment above the vacuum intensity, i.e., the intensity of one sheet. Data acquired from a number of experiments, carried out on different samples and different days, yielded the same results for the HAADF and the integrated EEL intensity.

The graphene plasmon behavior can be compared with 
that of thin carbon nanotubes, where the bulk plasmon subsides and only tangential and radial surface modes prevail. A trend has been observed for the $\sigma$ plasmon in tubes, where $r / R->1,{ }^{5}$ namely, that the tangential (or in plane) mode increases on the expense of the radial (or out of plane) and, looking at Fig. 3(a) in Ref. 5, where plasmon spectra for tubes with increasing $r$ are presented, it furthermore appears that for large $r$, only the tangential mode (at $\sim 15 \mathrm{eV}$ ) remains, very similar to the graphene case. However, although the resemblance with single-wall nanotube EEL data is large, it cannot, from the start, be assumed that the graphene low loss function reduces to the same theoretical approximation, namely, to that derived for an electron passing in aloof geometry. ${ }^{18}$ For large tube diameters, in the work by Taverna et al. ${ }^{18}$ the dielectric response tends to that of an anisotropic thin foil in the weak coupling regime, however, in their case, the electron passes parallel at a distance, whereas for a penetrating geometry, as in our case, the potential would have to be modified for electron transit within the foil. There has been no expression of the low loss function derived using dielectric theory and continuum models for the latter case. Here, we derive the microscopic loss function for this scenario via $a b$ initio calculations.

These deal with supercells composed of carbon layers. We imagine that the EELS experiment can be modeled by a monochromatic beam of electrons whose momentum transfer $\boldsymbol{q}$ to the sample is along either the $\boldsymbol{c}$ or $\boldsymbol{a}$ axis of the unit cell. The unit cell contains planes of carbon atoms, which are separated by multiples of the interlayer separation found in graphite. Thus, to model graphene, layers of carbon atoms are removed from the supercell leaving single layers well separated from each other. Bilayers and trilayers can be modeled by removing layers as before but leaving pairs of planes with the separation and stacking sequence found in graphite but each bilayer or trilayer is well separated from its neighbor.

The rate at which energy and momentum $\hbar q$ is lost from a charged particle moving through a homogeneous dielectric with speed $\boldsymbol{v}$ due to ionization is proportional to the loss function, which is the imaginary part of the inverse longitudinal dielectric constant $\varepsilon_{i j}$. ${ }^{19}$ Within the random phase approximation, ${ }^{20,21}$ the expression for $\varepsilon_{i j}$ is

$$
\varepsilon_{i j}(q, E)=\delta_{i j}+\left(\frac{e^{2}}{\varepsilon_{0} \Omega}\right) \sum_{k} \frac{(r \cdot i)_{k v, k+q c}(r \cdot j)_{k+q c, k v}}{E_{k+q, c}-E_{k, v}-E}
$$

Here, $\boldsymbol{E}_{\boldsymbol{k}, \boldsymbol{c}}$ and $\boldsymbol{E}_{\boldsymbol{k}, \boldsymbol{v}}$ denote the empty and filled bands and $\boldsymbol{\Omega}$ is the volume of the unit cell. The sum is over a special set of $\boldsymbol{k}$ vectors. For graphene and graphite, the principal values of the loss tensor lie along $\boldsymbol{c}$ and $\boldsymbol{a}$. This formulation includes the effect of multiple inelastic scattering but assumes a homogeneous system. We use the AIMPRO local density functional $\operatorname{code}^{22}$ to evaluate the imaginary part of the dielectric tensor for $\boldsymbol{q}=0$ and use the Kramers-Kronig relations to derive its real part. We use $43000 k$-points and a broadening of $0.5 \mathrm{eV}$ to fully converge the results. The loss function is then found for graphite, graphene, and bimultilayers and trimultilayers.
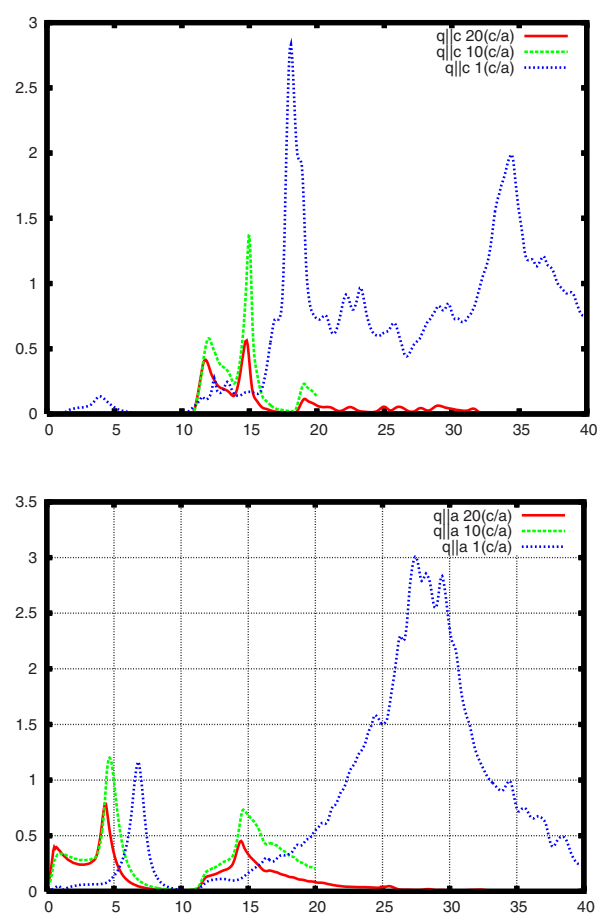

FIG. 2. (Color online) The loss function for graphite with a separation between $c$-planes equal to 20 and 10 times its separation in graphite and for graphite itself. Figure 2 (top) is case where $\boldsymbol{q} \| \boldsymbol{c}$ and Fig. 2 (bottom) when $\boldsymbol{q} \| \boldsymbol{a}$. $Y$ axis: arbitrary units, $x$ axis: energy loss in $\mathrm{eV}$.

We first consider graphite. The top and bottom panels of Fig. 2 show the loss function for $\boldsymbol{q}$ parallel to $\boldsymbol{c}$ and $\boldsymbol{a}$ (blue dotted-line spectra), respectively. We find that the former has peaks around $4,12,14$, and $18 \mathrm{eV}$ while the latter has a peak at $7 \mathrm{eV}$ and a broad peak at $27 \mathrm{eV}$. The 7 and $27 \mathrm{eV}$ peaks are due to $\pi$-electron plasmons (one electron per atom) and $\pi$ plus $\sigma$ (four electrons per atom) plasmons reflecting the vanishing of the real part of the dielectric constant.

The $\sim 4 \mathrm{eV}$ peak is due to a $\pi$ to $\pi^{*}$ transition. It is noteworthy that the height of the $\sim 4 \mathrm{eV}$ peak is about $10 \%$ of the height of the $7 \mathrm{eV}$ peak and, hence, the main plasmon loss below $10 \mathrm{eV}$ is due to the plasmon moving along $\boldsymbol{a}$.

These results are in reasonable agreement with studies of the $\boldsymbol{c}$ and $\boldsymbol{a}$ plasmons in graphite: ${ }^{23}$ Here, for $\boldsymbol{q}$ parallel to $\boldsymbol{c}$, peaks are observed at 4.6, 13, 15 , and $19 \mathrm{eV}$ but for $\boldsymbol{q}$ parallel to $\boldsymbol{a}$, only peaks at $6.8 \mathrm{eV}$ and a broad peak at $23 \mathrm{eV}$ are seen. Moreover, the intensity of the $4.6 \mathrm{eV}$ peak is about $20 \%$ that of the $6.8 \mathrm{eV}$ peak. This experiment shows that energy loss in a transmission experiment, such as used here, will be due to both $\boldsymbol{q}$ parallel to $\boldsymbol{a}$ and $\boldsymbol{q}$ parallel to $\boldsymbol{c}$ plasmons. The close agreement with the calculations suggests that the loss function $\operatorname{Im}(1 / \varepsilon)$ is an appropriate way of modeling the loss for planar sheets of graphite.

We now turn to graphene. This was modeled by expanding the lattice parameter along $\boldsymbol{c}$. The loss functions for $\boldsymbol{q}$ parallel to $\boldsymbol{c}$ and $\boldsymbol{a}$ for 10 and 20 fold expansions are shown in Fig. 2 as green dotted-line and red full-line spectra, respectively. In the latter case where $\boldsymbol{q}$ is parallel to $\boldsymbol{c}$ (top panel), the loss is almost zero up to $12 \mathrm{eV}$ (red line) and after this the onset occurs at a similar energy to graphite. However, the peak heights are very different from graphite. The 
disappearance of the $4 \mathrm{eV}$ peak can be explained by a selection rule. As stated above, the peak is due to a transition between occupied $\pi$ and unoccupied $\pi^{*}$ bands at the $M$ point of the Brillouin zone. Inspection of the wave functions for these two states shows that both transform as $\boldsymbol{p}_{z}$ and are odd under reflection symmetry present in the basal plane of graphene but not $\mathrm{AB}$ graphite and, hence, the dipole matrix element between them vanishes for transitions for which $\boldsymbol{q}$ is parallel to $c$ although the transition is allowed for $\boldsymbol{q}$ parallel to $\boldsymbol{a}$. This shows that the dielectric constant of graphene is not the same as graphite.

Figure 2, bottom panel, compares the loss function for $\boldsymbol{q}$ parallel to $\boldsymbol{a}$ for graphene and graphite. We note there are substantial redshifts of the peaks found in graphite. ${ }^{13}$ The 7 $\mathrm{eV} \pi$ plasmon peak has shifted downward to about $4.8 \mathrm{eV}$ while the broad peak around $27 \mathrm{eV}$ has sharpened and shifted to below $15 \mathrm{eV}$. Such shifts are seen in the experimental spectra shown in Fig. 1.

We now investigate the loss functions for bilayers and trilayers stacked as in graphite. The precise peak positions depend on the separation of planes and to compare the loss functions for graphite and multiple layers, we choose the separation between periodically repeated multilayers to be 5 times the separation in graphite. We also use a broadening of $1.5 \mathrm{eV}$. The supercell containing the trilayer for example, has three layers of graphene separated by the interlayer separation found in graphite, but the separation of these planes from similar planes in adjacent unit cells along the $c$ axis is now five times the separation in graphite. The resulting loss function is shown in Fig. 3 for single, double, and triple layers, as well as for graphite. It is clear that there is an increasing redshift of the peaks above, about $10 \mathrm{eV}$ as the number of layers decreases. The relative increase in amplitudes of peaks for the different layers (Fig. 3, bottom panel) seem roughly consistent with experimental spectra in Fig. 1 for one, two, and five layers but the observed spectra are broader. All the layers except the single one show the outof-plane plasmon peak around $4 \mathrm{eV}$.

In conclusion, the $4.6 \mathrm{eV}$ out-of-plane loss peak found in graphite disappears for a single layer graphene. This is related to a selection rule operating in graphene but not in graphite. This, however, does not occur for in-plane modes or a graphene bilayer with $\mathrm{AB}$ stacking but does also occur
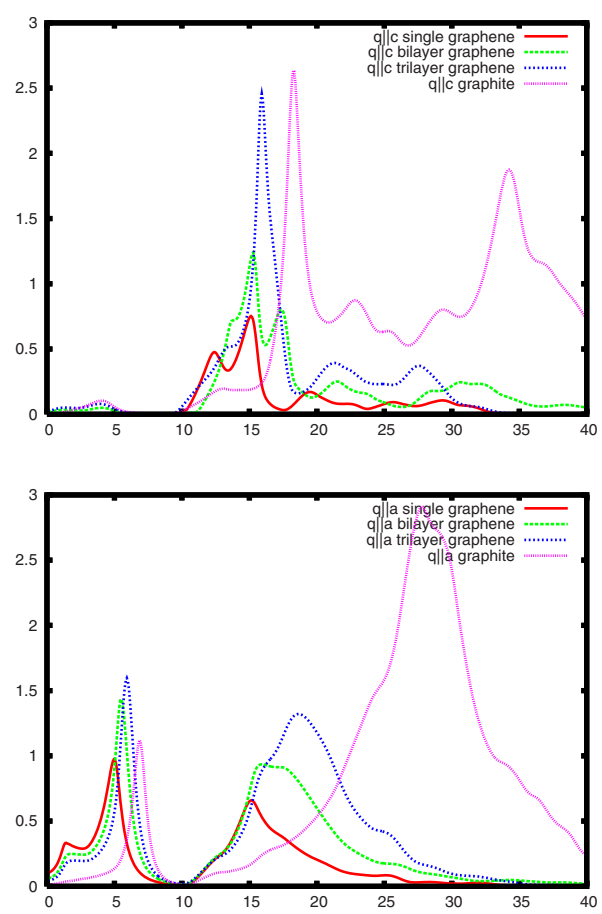

FIG. 3. (Color online) Comparison of the loss function for a single and multilayers of graphene for $\boldsymbol{q} \| \boldsymbol{c}$ (top) and $\boldsymbol{q} \| \boldsymbol{a}$ (bottom). Note, the loss around $4 \mathrm{eV}$ in the $\boldsymbol{q} \| \boldsymbol{c}$ case for the multilayer case and its absence for single layer graphene (shown as red line). Note also the increasing redshift of the main peaks above about $10 \mathrm{eV}$ as the number of layers decreases. $Y$ axis: arbitrary units, $x$ axis: energy loss in $\mathrm{eV}$.

for a bilayer with AA stacking. It implies that any observations of a loss below $10 \mathrm{eV}$ due to these plasmons must be due to adsorbates lying on graphene and makes graphene peculiarly sensitive to such adsorbates. We note that these plasmons could be excited by light of grazing incidence and polarized along $c$. A further feature, which is unique to the plasmon behavior of graphene, is the shift of the $7 \mathrm{eV}$ inplane plasmon seen in graphite to about $4.7 \mathrm{eV}$, as well as a substantial redshift of the broad plasmon peak at $25 \mathrm{eV}$, seen in graphite to about $14.6 \mathrm{eV}$ in graphene accompanied by a distinct shape change to skewed triangular. The redshifts decrease as the number of close-by layers increases.
${ }^{1}$ K. S. Novoselov et al., Science 306, 666 (2004).

${ }^{2}$ A. K. Geim and K. S. Novoselov, Nat. Mater. 6, 183 (2007).

${ }^{3}$ F. Schedin et al., Nat. Mater. 6, 652 (2007).

${ }^{4}$ C. J. Meyer et al., Nature (London) 446, 60 (2007).

${ }^{5}$ O. Stéphan et al., Phys. Rev. B 66, 155422 (2002).

${ }^{6}$ H. Raether, Phys. Thin Films 9, 149 (1977).

${ }^{7}$ A. Otto, Phys. Status Solidi 22, 401 (1967).

${ }^{8}$ J. F. Annett et al., Phys. Rev. B 37, 2408 (1988).

${ }^{9}$ P. Laitenberger and R. E. Palmer, Phys. Rev. Lett. 76, 1952 (1996).

${ }^{10}$ R. Perez and W. Que, J. Phys.: Condens. Matter 18, 3197 (2006).

${ }^{11}$ M. Kociak et al., Phys. Rev. Lett. 87, 075501 (2001).

${ }^{12}$ E. H. Hwang and S. Das Sarma, Phys. Rev. B 75, 205418
(2007).

${ }^{13}$ A. G. Marinopoulos et al., Appl. Phys. A: Mater. Sci. Process. 78, 1157 (2004).

${ }^{14}$ A. Nagashima et al., Solid State Commun. 83, 581 (1992).

${ }^{15}$ R. R. Nair et al., Science (to be published).

${ }^{16} \mathrm{U}$. Bangert et al. (unpublished).

${ }^{17}$ M. H. Gass et al. (unpublished).

${ }^{18}$ D. Taverna et al., Phys. Rev. B 66, 235419 (2002).

${ }^{19}$ P. Nozieres and D. Pines, Phys. Rev. 113, 1254 (1959).

${ }^{20}$ H. Ehrenreich and M. H. Cohen, Phys. Rev. 115, 786 (1959).

${ }^{21}$ S. L. Adler, Phys. Rev. 126, 413 (1962).

${ }^{22}$ U. Bangert et al., Philos. Mag. 86, 4757 (2006).

${ }^{23}$ K. Zeppenfeld, Phys. Lett. 25A, 335 (1967). 